\title{
VLT observations of the central compact object in the Vela Jr. supernova remnant ${ }^{\star}$
}

\author{
R. P. Mignani ${ }^{1}$, A. De Luca ${ }^{2}$, S. Zaggia ${ }^{3}$, D. Sester ${ }^{3}$, A. Pellizzoni ${ }^{2}$, S. Mereghetti ${ }^{2}$, and P. A. Caraveo ${ }^{2}$ \\ 1 Mullard Space Science Laboratory, University College London, Holmbury St. Mary, Dorking, Surrey, RH5 6NT, UK \\ e-mail: rm2@mssl.ucl.ac.uk \\ INAF, Istituto di Astrofisica Spaziale, via Bassini 15, Milan 20133, Italy \\ e-mail: [deluca; sester; alberto; sandro;pat]@iasf-milano.inaf.it \\ 3 INAF, Osservatorio Astronomico di Padova, Vicolo dell'Osservatorio 5, Padua 35122, Italy \\ e-mail: simone.zaggia@oapd.inaf.it
}

Received 1 May 2007 / Accepted 3 July 2007

\begin{abstract}
Context. X-ray observations have unveiled the existence of enigmatic point-like sources at the center of young (a few kyrs) supernova remnants. These sources, known as central compact objects (CCOs), are thought to be neutron stars produced by the supernova explosion, although their X-ray phenomenology makes them markedly different from all other young neutron stars discovered to date. Aims. The aim of this work is to search for the optical/IR counterpart of the Vela Junior CCO and to understand the nature of the associated $\mathrm{H}_{\alpha}$ nebula discovered by Pellizzoni et al. (2002).

Methods. We used deep optical ( $R$-band) and IR $\left(J, H, K_{\mathrm{s}}\right.$-bands) observations recently performed by our group with the ESO VLT to obtain the first deep, high resolution images of the field to resolve the nebula structure and pinpoint a point-like source possibly associated with the neutron star.

Results. Our $R$-band image shows that the nebula's flux and structure are very similar to those of, the $\mathrm{H}_{\alpha}$ one, suggesting that the nebula spectrum is dominated by pure $\mathrm{H}_{\alpha}$ line emission. However, the nebula is not detected in our IR observations, which makes it impossible to to constrain its spectrum. A faint point-like object $\left(J \geq 22.6, H \sim 21.6, K_{\mathrm{s}} \sim 21.4\right)$ compatible with the neutron star's Chandra X-ray position is detected in our IR images ( $H$ and $K_{\mathrm{s}}$ ) but not in the optical image $(R \gtrsim 25.6)$, where it is buried by the nebula background.

Conclusions. The nebula is most likely a bow-shock produced by the neutron star motion through the ISM or, alternatively, a photoionization nebula powered by UV radiation from a hot neutron star. A synchrotron nebula, powered by the relativistic particle wind from the neutron star, is the less likely interpretation because of its non-detection in the X-rays and the apparent lack of continuum emission. The candidate CCO counterpart could be the neutron star itself, a fallback disk left over from the supernova explosion, or a mid M-type star, or later, at a distance of $2 \mathrm{kpc}$.
\end{abstract}

Key words. stars: neutron - X-rays: individuals: CXO J085201.4-461753 - ISM: supernova remnants

\section{Introduction}

Ground breaking observations with the current generation of $\mathrm{X}$-ray observatories have recently unveiled unexpected and puzzling aspects of the phenomenology of isolated neutron stars (INSs). It has become clear that not all INSs are born as fast spinning, active radio pulsars. A handful of isolated compact sources, most probably young INSs, do not show any "standard" pulsar activity. They are known as "central compact objects" (CCOs) in supernova remnants (Pavlov et al. 2004), enigmatic compact sources lying very close to the center of young (0.3-20 kyrs) supernova remnants, showing only thermal-like often unpulsed X-ray emission. They are thought to be the youngest members of the radio-quiet NS family which also includes the anomalous X-ray pulsars (AXPs) and the soft gammaray repeaters (SGRs) which make up the class of the magnetars (Woods \& Thompson 2006), the INSs with X-ray dim thermal emission (XDINSs, Haberl 2005), as well as high-energy pulsars such as Geminga (Caraveo et al. 1996). At present, we cannot

* Based on observations collected at the European Southern Observatory, Paranal, Chile under programme ID 074.D-0729(A), 077.D-0764(A). exclude that such sources represent "the rule" among INSs, radio pulsars being "the exception", but are more easily observed. Their overall properties are largely unknown. First, the lack of observable radio emission still has to be explained. It could be possibly due to an unfavorable beaming, to inhibition by an ultra-high magnetic field, as invoked for the magnetars, or to quenching by accretion from a residual disk. The nature of the CCO thermal X-ray emission is also unclear. It could be produced by the cooling NS surface, by hot polar caps, by the NS magnetic field decay, or by accretion from a residual disk. Their relationship (if any) with other classes of radio-quiet INSs is also debated.

As in the case of other radio-quiet INSs, optical/IR observations could provide a major step forward in the understanding of the nature of the CCOs. Sanwal et al. (2002) identified a field $\mathrm{M}$ dwarf detected by $H S T$ as a likely IR counterpart to the RCW103 CCO, possibly making it the first X-ray Binary associated with a SNR. The identification, however, is still unconfirmed. IR observations of other CCOs have recently been carried out (Fesen et al. 2006; Wang et al. 2007), but no viable candidate counterpart has been identified. An IR counterpart to the PKS 1209-52 CCO was proposed by Pavlov et al. (2004) but 
the identification was recently discarded both by Mignani et al. (2007a) and Wang et al. (2007) on the basis of its significant offset with respect to the Chandra position. The unsuccessful identification score is explained by the fact that most CCOs are rather distant and heavily absorbed, and therefore are very difficult targets at optical/IR wavelengths.

The only possible exception is the X-ray source at the center of the RXJ 0852.0-4622 supernova remnant, and one of the least studied CCOs. RXJ 0852.0-4622 (G266.1-1.2) is a very young (a few thousands years) shell-like supernova remnant discovered in the ROSAT All Sky Survey (Aschenbach 1998), whose projected position is coincident with the southeast edge of the more extended Vela supernova remnant, hence dubbed "Vela Junior". However, its estimated distance of $\sim 1 \mathrm{kpc}$ (Slane et al. 2001) puts it beyond the Vela supernova remnant and thus rules out any intriguing association between the two. The CCO in Vela Jr. (AX J0851.9-4617.4) was discovered by ASCA (Slane et al. 2001) and studied with BeppoSax (Mereghetti 2001) and later with Chandra, which also provided its sub-arcsec position (Pavlov et al. 2001). The X-ray emission of the Vela Jr. CCO, hereafter CXO J085201.4-461753, is characterized by a thermallike spectrum with $k T \approx 404 \mathrm{eV}\left(N_{\mathrm{H}} \approx 3.5 \times 10^{21} \mathrm{~cm}^{-2}\right)$ and an emitting radius of $\approx 0.28 \mathrm{~km}$ (at $1 \mathrm{kpc}$ ), with no evidence of pulsations (Kargaltsev et al. 2002). The first optical investigations of the CXO J085201.4-461753 field were carried out by Pavlov et al. (2001) with the CTIO $0.9 \mathrm{~m}$ telescope, and by Mereghetti et al. (2002) using archival observations obtained with the ESO/MPG $2.2 \mathrm{~m}$ telescope. The derived optical flux upper limits of $B \sim 23$ and $R \sim 22.5$ (Mereghetti et al. 2002) imply a very high $\mathrm{X}$-ray to optical flux ratio which virtually certified CXO J085201.4-461753 as the compact remnant of the Vela Jr. supernova explosion. Although no optical candidate counterpart was detected by Mereghetti et al. (2002), a short $\mathrm{H}_{\alpha}$ exposure unveiled the presence of an extended emission feature $\left(\sim 6^{\prime \prime}\right.$ diameter) positionally compatible with the CXO J085201.4-461753 error circle. The reality of this feature was confirmed soon after by Pellizzoni et al. (2002) using a deeper $\mathrm{H}_{\alpha}$ exposure $(\sim 3 \mathrm{~h})$ taken as a part of the Southern Galactic Plane and Magellanic Clouds digitized plate survey (Parker \& Phillips 1998) carried out with the $A A O$ UK Schmidt Telescope. Unfortunately, the low spatial resolution of the $\mathrm{H}_{\alpha}$ image ( $1^{\prime \prime} /$ pixel) did not allow the resolution of the feature structure and the selection of one of the two alternative proposed interpretations i.e., a velocity-driven bow-shock nebula, or a photo-ionization nebula, powered by UV radiation from a hot INS, therefore better constraining the properties of the CCO.

Here we present the results of the first deep optical and IR observations of the Vela Jr. CCO recently performed with the ESO VLT. Optical and IR observations are presented in Sects. 2 and 3, respectively and the results are discussed in Sect. 4.

\section{Optical observations}

\subsection{Observations description}

We performed deep optical observations of the Vela Jr. CCO with FORS1 (FOcal Reducer Spectrograph), a multi-mode instrument for imaging and long-slit/multi-object spectroscopy mounted at the second Unit Telescope (UT2) of the VLT (Paranal Observatory). The observations were carried out in Service Mode on January 15th and 17th 2005 through the $R$ Bessel filter $(\lambda=6570 \AA ; \Delta \lambda=1500 \AA)$. The four-port readout mode and high gain were choosen as the default instrument configuration. To minimize light pollution from "star Z" of
Table 1. Summary of the VLTFORS1 R-band observations of the Vela Jr. field with the number of exposures per night, the single exposure time, the average seeing and airmass values.

\begin{tabular}{lllll}
\hline \hline yyyy.mm.dd & $N$ & Exp. (s) & Seeing & Airm. \\
\hline 2005.01 .15 & 10 & 259 & 0.93 & 1.3 \\
2005.01 .17 & 10 & 259 & 0.93 & 1.3 \\
\hline
\end{tabular}

Pavlov et al. (2001), located $\sim 1$ 1"5 from our target, we split the integration time in 20 exposures of $260 \mathrm{~s}$ each. In order to achieve the best possible spatial resolution, useful to disentangle any point source contribution from the PSF wings of star Z, FORS 1 was used in its high resolution $(\mathrm{HR})$ mode $(1 \times 1$ binning), with a corresponding pixel size of $0 . ' 1$. The very bright stars HD $76060(V=7.88)$ and Wray 16-30 $(V=13.8)$, respectively $\approx 35^{\prime \prime}$ and $\approx 25^{\prime \prime}$ from our target (Mereghetti 2001), were masked using the FORS1 occulting bars. The observations were collected with an average seeing of $\sim 0{ }^{\prime} 8-00^{\prime} 9$ and an airmass $\sim 1.3$ (Table 1 ) on both nights and with a fractional lunar illumination of $57 \%$ and $47 \%$ for the first and second night, respectively.

\subsection{Data reduction and analysis}

Usual reduction steps to remove instrumental signatures were applied through the ESO FORS1 data reduction pipeline ${ }^{1}$ using bias frames and twilight flat fields. Single reduced images were then combined to filter out cosmic rays. Since no dither was applied between single exposures to preserve the masking of the bright stars in the field, no image registration was required before the frame stacking. Photometric calibration was performed through the observation of standard stars from the field PG132386 (Landolt 1992), yielding $R$-band zeropoints of $26.86 \pm 0.05$ and $27.48 \pm 0.03$ for the first and second night, respectively. We note that the first night was not photometric, with the computed zeropoint deviating by $\sim 0.6 \mathrm{mag}$ from the trend ${ }^{2}$. Thus, we re-normalized our photometry using as a reference the zeropoint of the second night which, instead, is very well consistent with the zeropoint trend. The coordinates of the Vela Jr. CCO were measured with a good nominal accuracy $\left(0{ }^{\prime} 6\right)$ by Pavlov et al. (2001) who report: $\alpha(J 2000)=08^{\mathrm{h}} 52^{\mathrm{m}} 01.38^{\mathrm{s}}, \delta(J 2000)=$ $-46^{\circ} 17^{\prime} 53^{\prime \prime} 34$. We re-analyzed the same Chandra observation and obtained: $\alpha(J 2000)=08^{\mathrm{h}} 52^{\mathrm{m}} 01.37^{\mathrm{s}}, \delta(J 2000)=$ $-46^{\circ} 17^{\prime} 53^{\prime \prime} 50$, i.e. with a slight offset southwest but statistically consistent with previous reports.

The astrometry on the FORS1 image was computed using as a reference 26 stars selected from the $2 M A S S$ catalogue. The pixel coordinates of these stars (all non saturated and evenly distributed in the field) were measured by Gaussianfitting their intensity profiles using the specific function of the GAIA (Graphical Astronomy and Image Analysis) tool ${ }^{3}$ while the fit to the RA, Dec reference frame was performed using the Starlink package ASTROM ${ }^{4}$. The rms of the astrometric solution was $\approx 0$.' 12 per coordinate. After accounting for the 0.2 average astrometric accuracy of the $2 M A S S$, the overall uncertainty to be attached to the position of our target is 0.65 .

A $8^{\prime \prime} \times 8^{\prime \prime}$ zoom of the FORS1 R-band image of the field is shown in Fig. 1-left. No point-like source appears at the Chandra

\footnotetext{
1 wWw. eso.org/observing/dfo/quality/FORS1/pipeline

2 www .eso.org/observing/dfo/quality/FORS1/qc/ zeropoints

3 star-www.dur.ac.uk/ pdraper/gaia/gaia.html

4 http://star-www.rl.ac.uk/Software/software.htm
} 


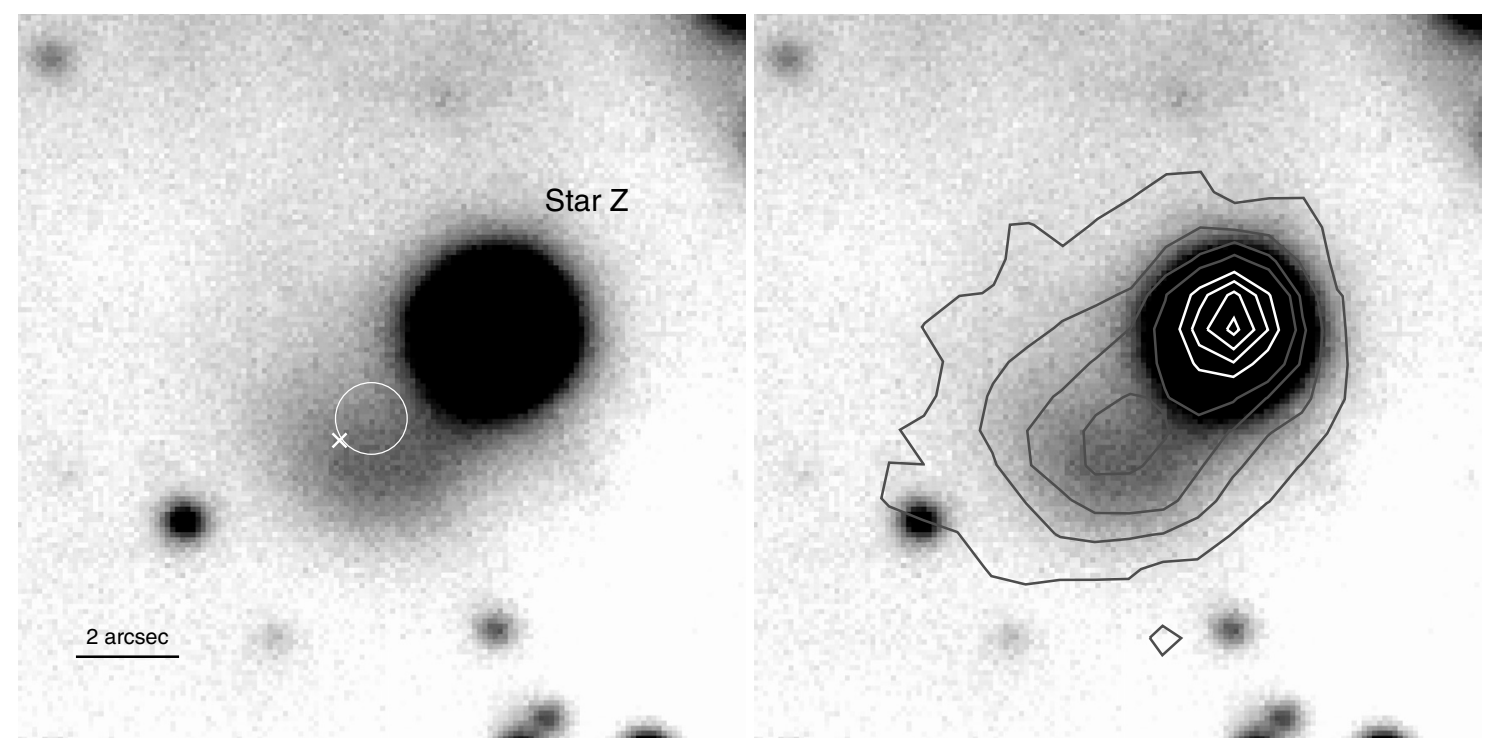

Fig. 1. (Left) Inner section $\left(8^{\prime \prime} \times 8^{\prime \prime}\right)$ of the VLT/FORS1 R-band image of the Vela Jr. CCO. North to the top, East to the left. The circle $(0.7$ radius, $1 \sigma$ ) corresponds to the recomputed Chandra position uncertainty after accounting for the accuracy of our astrometric solution (see Sect. 2.2). Star Z of Pavlov et al. (2001) is labelled. The cross indicates the position of the candidate CCO counterpart (see Sect. 3.3). (Right) Isophotal contours from the UKST $\mathrm{H}_{\alpha}$ image of Pellizzoni et al. (2002) overplotted. Dark and light isophotes have a linear and geometric spacing in intensity, respectively. The coincidence of the diffuse feature both in position and in morphology is evident.

position, thus setting an upper limit of $R \sim 25.6(3 \sigma)$ on the CCO counterpart i.e., about 3 mag deeper than that obtained by Mereghetti et al. (2002). Instead, a compact optical nebula is cleary detected. We exclude that this nebula is an artifact due to a PSF anomaly of star Z, to a defect in the image flat fielding, to ghost images, or to any other instrumental effect. Both its position and extent (Fig. 1-right), are consistent with that of the $\mathrm{H}_{\alpha}$ nebula seen by Pellizzoni et al. (2002), which clearly indicates that they are the same object. The nebula brightness in the $R$-band is $\sim 22.8 \mathrm{mag} \mathrm{arcsec}^{-2}$ in the central part. Unfortunately, no color information is available on the nebula and thus, it is not possible to constrain its spectrum. However, we note that the $R$-band flux of the nebula is $(3.7 \pm 0.3) \times 10^{-14} \mathrm{erg} \mathrm{cm}^{-2} \mathrm{~s}^{-1}$, which is consistent with the value of $(3.0 \pm 0.6) \times 10^{-14} \mathrm{erg} \mathrm{cm}^{-2} \mathrm{~s}^{-1}$ measured in $\mathrm{H}_{\alpha}$ by Pellizzoni et al. (2002). This suggests that the nebula spectrum has, at least in the $R$-band, no significant continuum components and is dominated by pure $\mathrm{H}_{\alpha}$ line emission.

\section{IR observations}

\subsection{Observations description}

Deep IR observations of the Vela Jr. CCO were performed in visitor mode on May 23rd and 24th 2006 with NAos COnica $(N A C O)$, an adaptive optics (AO) imager and spectrometer mounted at the fourth Unit Telescope (UT4) of the VLT. To provide the best combination between angular resolution and sensitivity, NACO was operated with the S27 camera with a corresponding field of view of $28^{\prime \prime} \times 28^{\prime \prime}$ and a pixel scale of 0 . $^{\prime} 027$. As a reference for the AO correction we used the GSC-2 star S1331311130291 ( $V=15.3)$, located 11'.3 from our target. The Visual (VIS) dichroic element and wavefront sensor (4500-10000 $\AA$ ) were used. Observations were performed in the $J(\lambda=12650 \AA ; \Delta \lambda=2500 \AA), H(\lambda=16600 \AA$; $\Delta \lambda=3300 \AA)$ and $K_{\mathrm{s}}(\lambda=21800 \AA ; \Delta \lambda=3500 \AA)$ filters, with a total net integration time of about $2300 \mathrm{~s}$ per band. To allow for subtraction of the variable IR sky background, each integration was split into sequences of short randomly dithered exposures
Table 2. Summary of the VLTNACO J, $H, K$-band observations of the Vela Jr. field with the number of exposures per filter $(N \times$ NDIT $)$, the Detector Integration Time (DIT), the average seeing and airmass values.

\begin{tabular}{lcllll}
\hline \hline yyyy.mm.dd & Fil. & $N \times$ NDIT & DIT $(\mathrm{s})$ & Seeing & Airm. \\
\hline 2006.05 .23 & $K_{\mathrm{s}}$ & $5 \times 19$ & 24 & $0 \prime^{\prime} 78$ & 1.18 \\
& $H$ & $2 \times 19$ & 60 & 0.81 & 1.32 \\
2006.05 .24 & $K_{\mathrm{s}}$ & $2 \times 20$ & 60 & $0 !^{\prime} 74$ & 1.19 \\
& $J$ & $2 \times 21$ & 60 & $0 !^{\prime} 63$ & 1.25 \\
\hline
\end{tabular}

with Detector Integration Times (DIT) of 24 and $60 \mathrm{~s}$ and NDIT repetitions along each point of the dithering pattern (see Table 2). The instrument readout mode was selected according to the DIT to minimize the readout noise. For all observations, the seeing conditions were on average below $\sim 0$ ' 8 and the airmass was better than 1.3, allowing optimal use of the NACO adaptive optics. Unfortunately, the AO correction degraded significantly towards the end of the $H$-band exposure sequence on the first night. Night (twilight flat fields) and day time calibration frames (darks, lamp flat fields) were taken daily as part of the NACO calibration plan. Standard stars from the Persson et al. (1998) fields were observed on both nights for photometric calibration.

\subsection{Data reduction and analysis}

Data were processed using the ESO NACO pipeline $^{5}$ and the science images reduced with the produced master dark and flat field frames. For each band, single reduced science exposures were then combined by the NACO pipeline to produce cosmicray free and sky-subtracted images. The photometric calibration pipeline yielded average zeropoints of $23.87 \pm 0.07(J)$, $23.81 \pm 0.05(H), 22.93 \pm 0.03\left(K_{\mathrm{s}}\right)$ for the first night and $23.98 \pm 0.04(J), 23.85 \pm 0.04(H), 22.85 \pm 0.03\left(K_{\mathrm{s}}\right)$ for the second night.

The astrometric calibration of the NACO images was performed using the same procedure described in Sect. 2.2.

${ }^{5}$ www.eso.org/observing/dfo/quality/NACO/pipeline 


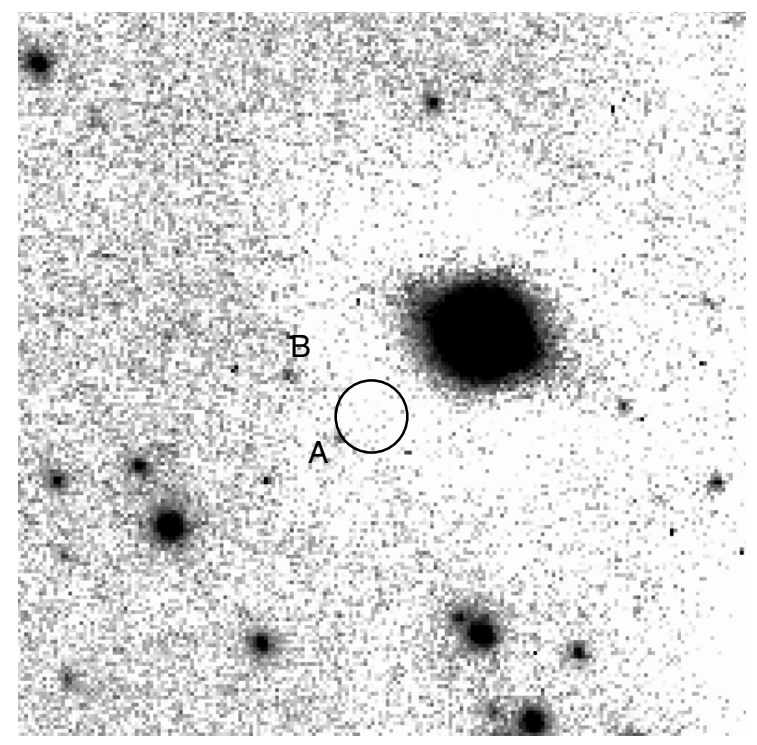

Fig. 2. $14^{\prime \prime} \times 14^{\prime \prime}$ section of the VLT/NACO co-added $J H K_{\mathrm{s}}$-band image of the Vela Jr. CCO. North to the top, East to the left. The circle ( 0.7 radius) corresponds to the Chandra position uncertainty after accounting for the accuracy of our astrometric solution (see Sect. 3.2). Star Z of Pavlov et al. (2001) is visible northwest of the error circle. The faint object $\left(H=21.6 \pm 0.1 ; K_{\mathrm{s}}=21.4 \pm 0.2\right)$, labelled A, close to the southeast edge of the error circle is the possible IR counterpart of the CCO. Another faint object $(J=21.2 \pm 0.2)$, labelled $\mathrm{B}$, is visible $\sim 1^{\prime \prime} 9$ northeast from the computed Chandra position.

However, since only four $2 M A S S$ stars are identified in the narrow NACO S27 field of view, we have computed our astrometry using as a reference a set of ten secondary stars found in common with our FORS1 $R$-band image, calibrated using $2 M A S S$ (Sect. 2.2). The rms of the astrometric solution was $\approx 0$ '.06 per coordinate. By adding in quadrature the rms of the astrometric solution of the FORS1 image ( $\approx 0 . ' 12$ per coordinate) and after accounting for the astrometric accuracy of $2 M A S S$ we obtained an overall accuracy of 0.37 per coordinate on the NACO images astrometry. Assuming the same Chandra positional error as before $\left(0{ }^{\prime} 6\right)$, the overall uncertainty on the target position on the $N A C O$ images is thus 0.7 .

\subsection{Results}

We identified a very faint, apparently point-like object close to the southeast edge of the Chandra CCO error circle (Fig. 2). Although it is detected with a marginal significance $(\sim 4 \sigma)$, we exclude that this object is a fake detection due to a background fluctuation, a detector feature, or a data reduction artifact. Its reality is confirmed by its independent detection in both the $K_{\mathrm{s}}$-band images taken on the two consecutive nights as well as in the $H$-band image. However, the object is not detected in the $J$-band image and in the FORSI $R$-band one $(R \gtrsim 25.6)$, where it is buried by the nebula background (Sect. 2.3). Since the NACO PSF is largely oversampled, we resampled all the reduced $J, H$ and $K_{\mathrm{s}}$-band images to obtain a detection with a better signalto-noise ratio. We used the swarp program (Bertin E., Terapix Project) to resample the $N A C O$ images with a $3 \times 3$ pixels window while conserving the flux. This helped to reduce the noise and enhance the faintest objects. Resampled images were then registered and co-added. Figure 2 shows the co-added $J H K_{\mathrm{s}}$-band image with the computed Chandra position of the CCO overlaid. The object close to the Chandra error circle is labeled A.
A second point-like object (labeled B) is identified in the coadded image, although it is detected in the $J$-band image only, $\sim 1$ '.9 northeast from the computed Chandra position. However, the almost $2 \sigma$ offset from the edge of the error circle suggests that it is likely to be a background object. We thus assume that object A is the only possible counterpart to the Vela Jr. CCO.

We computed the magnitudes of object A using PSF photometry. Although the accuracy of the PSF photometry certainly depends on the AO correction, the target faintness makes PSF photometry more suitable than standard aperture photometry. We used the Stetson $(1992,1994)$ suite of programs Daophot, following the procedures outlined in Zaggia et al. (1997). As a reference for our photometry we have used the resampled $J, H$ and $K_{\mathrm{s}}$-band images, which have a much better signal-to-noise ratio. For each image we then calculated the model PSF with Daophot using a 6 parameters Penny function to fit the profile of the $\sim 15$ brightest non-saturated objects in the field. The model PSF was then used to measure the fluxes of object A as well as of all other objects in the field. To improve the precision of our PSF photometry, and to maximize the number of objects identified in the field, we used the Allframe routine of Daophot. From the co-added $J H K_{\mathrm{s}}$-band image we ran Daophot to create a master list of objects which we have properly registered on each single band image and then passed to Allframe to be used as a reference for PSF photometry in the single band images. The single band photometry catalogues were then matched by Allframe to create the final multi-band photometry catalogue. Photometry measurements obtained in the two $K_{\mathrm{s}}$-band images were averaged. We performed the absolute photometry calibration of our PSF photometry using the zeropoints provided by the NACO data reduction pipeline. Since the NACO zeropoints are computed using aperture photometry, the magnitudes computed by Daophot were corrected by applying the aperture correction. We finally applied the airmass correction using the atmospheric extinction coefficients for the Paranal Observatory. The magnitudes of object $\mathrm{A}$ then turn out to be $H=21.6 \pm 0.1$ and $K_{\mathrm{s}}=21.4 \pm 0.2$. Apart from object B $(J=21.2 \pm 0.2)$, no other object is detected within/close to the Chandra error circle down to $J \sim 22.6$, $H \sim 22.5$ and $K_{\mathrm{s}} \sim 21.8$.

\section{Discussion}

\subsection{The nebula}

Clumpy emission structures and filaments produced by shocks from the supernova explosion are detected in $\mathrm{H}_{\alpha}$ in young SNRs, normally (but not only) along the external rims. Thus, it is possible, although very unlikely, that the CCO projected position is by chance coincident with one of these structures. More likely, the nebula is produced by the neutron star ionization of the neutral Hydrogen in the SNR. We note that the existence of neutral Hydrogen in young SNRs, following atom recombination after the supernova explosion, has been confirmed in a number of cases (e.g. Michael 2000).

One of the possible interpretations of the nebula (Pellizzoni et al. 2002) is in terms of a bow-shock produced by the neutron star motion through the ISM. Interestingly, the $R$-band image shows for the first time that the nebula is not spherically symmetrical but looks more like a kidney bean, a shape somehow reminescent of the arc-like structures observed around bowshocks. Unfortunately, the spatial resolution of the VLT image is not sufficient to resolve more clearly the nebula morphology and to map its surface brightness distribution which, in the case of a velocity-driven bow-shock, would present a sharp enhancement 
towards the apex, depending on the actual value and direction of the neutron star velocity. We note that the position of the CCO lies behind the apparent apex of the nebula and roughly on its projected axis of symmetry, right where expected for a velocitydriven bow-shock. If object A were indeed the CCO counterpart, its position would be quite close to the nebula and slightly displaced from its apparent projected axis of symmetry. However, given the poor characterization of the nebula morphology and the lack of information on the neutron star spatial velocity, this would not affect the validity of the bow-shock interpretation.

Since proper motion measurements are not yet available for the Vela Jr. CCO, the interpretation can not be directly confirmed. No counterpart of the putative bow-shock has been detected so far in radio or in X-rays. However, we note that the detection of a bow-shock in $\mathrm{H}_{\alpha}$ only would not be in contradiction with its non-detection at other wavelengths. Indeed, the detection of a bow-shock in $\mathrm{H}_{\alpha}$ is typically anti-correlated with its detection in radio and in X-rays, with the only noticeable exception of the bow-shock found around the "Black Widow" pulsar (Stappers et al. 2003). A possible argument against the bow-shock interpretation comes from the position of the CCO with respect to the estimated geometrical center of the Vela Jr. SNR. According to Aschenbach (1998), this should be located at $\alpha(J 2000)=08^{\mathrm{h}} 52^{\mathrm{m}} 03^{\mathrm{s}}, \delta(J 2000)=-46^{\circ} 22^{\prime}$, which is $\sim 4$ arcmin south of the $\mathrm{CCO}$ position. This would imply a proper motion direction different from that expected from the apparent morphology of the nebula, which would be more compatible with a proper motion vector pointing southwest. However, both the uncertainty in the definition of the geometrical center of the SNR, e.g. the representative case of the Vela SNR (Aschenbach et al. 1995), and the still poorly characterized morphology of the nebula do not make this a strong argument to rule out the bowshock interpretation.

An alternative possibility is that the nebula around the Vela Jr. CCO is a photo-ionization nebula, powered by the UV radiation from the hot neutron star. The possible structure of a photo-ionization nebula associated with a neutron star moving supersonically through the interstellar medium was studied by Van Kerkwijk \& Kulkarni (2001). According to their model, a velocity-driven morphology is expected, with a definite symmetry with respect to the direction of the projected space velocity of the NS. Such a nebula should also have a smooth brightness profile, rather different from that of a bow-shock. Our VLT $R$-band image points to a morphology which could be consistent with such a picture. However, as for the case of the bow-shock scenario, any firm conclusion is prevented by the insufficient sharpness of the angular resolution of the ground-based images.

Although most of the nebula emission is in $\mathrm{H}_{\alpha}$ (Sect. 2.2), we cannot a priory rule out the possibility of a synchrotron nebula, powered by the relativistic particle wind emitted by the neutron star. These pulsar-wind nebulae are often observed around young rotation-powered pulsars, usually in the radio and in the $\mathrm{X}$-ray bands but also in the optical band. Bright optical pulsarwind nebulae have indeed been observed around the Crab pulsar (e.g. Hester et al. 1995) and PSR B0540-69 (Caraveo et al. 2001). However, while these objects also feature X-ray pulsarwind nebulae, with structures and morphologies very similar to the optical ones, no evidence for an X-ray nebula has been found for the Vela Jr. CCO. High-resolution Chandra X-ray observations (Pavlov et al. 2001) shows that the source profile is consistent with the instrument's PSF, which rules out the presence of an extended emission component, unless it is extremely faint. Recent radio observations performed with the Australian Telescope Compact Array (Reynoso et al. 2006) also ruled out the presence of a pulsar-wind nebula in radio. Thus, the lack of an X-ray/radio counterpart, together with the apparent lack of continuum optical emission, makes the pulsar-wind nebula interpretation for the Vela Jr. nebulosity very unlikely.

\subsection{The point source}

At the moment we can not confidently rule out the possibility that object $\mathrm{A}$ is just a field object unrelated to the $\mathrm{CCO}$, whose IR counterpart would thus remain unidentified. Indeed, given the density of stars in the NACO field of view we estimate a chance coincidence probability of $\sim 15 \%$, i.e. certainly not negligible. Given our flux upper limits $(R \sim 25.6, J \sim 22.6, H \sim 22.5$ and $\left.K_{\mathrm{s}} \sim 21.8\right)$, this would almost certainly rule out a hypothetical stellar companion (see discussion below) and would instead support the conclusion that the $\mathrm{CCO}$ is an isolated neutron star, possibly surrounded by a circumstellar disk. However, if object A is indeed the CCO counterpart, its nature is controversial. Indeed, it is not a priori obvious whether the detected IR emission can be ascribed to the neutron star itself, to a fallback disk around the neutron star, or to a putative companion star. Suggestive as it could be, the object does not show evidence for variability, with the $K_{\mathrm{s}}$ magnitudes measured in the two nights being consistent within the associated errors.

The first possibility is that object A is itself the IR counterpart of the neutron star. So far, IR emission has been detected for only five rotation-powered pulsars (see Mignani et al. 2007b, for a summary) and is ascribed to synchrotron radiation produced by relativistic particles in the neutron star's magnetosphere. The observed IR luminosity spans about six decades and is strongly dependent on the neutron star's age. If we assume for the Vela Jr. CCO an age of a few thousands years, as estimated for the host SNR, it might then have an IR luminosity comparable to that of the Vela pulsar, i.e. $\sim 10^{28} \mathrm{erg} \mathrm{s}^{-1}$. At a distance of $1 \mathrm{kpc}$ it would then have a magnitude $K_{\mathrm{s}} \sim 24.1$, i.e. much fainter than that of object A. IR emission from an isolated neutron would thus be incompatible with our detections unless the CCO distance is as low as $\sim 300 \mathrm{pc}$, or its IR luminosity is a factor $\sim 10$ higher than expected. However, given both the uncertainties on the $\mathrm{CCO}$ distance and the lack of information on its energetics, we can not a priori exclude that object $\mathrm{A}$ is itself the counterpart of the neutron star.

An alternative possibility is that the IR emission from object $A$ is produced from a fallback disk around the neutron star. Fallback disks have also been invoked to explain the IR spectral flattening of the AXPs (e.g., Israel et al. 2003, 2004) and have been searched around other objects possibly related to the magnetars, such as the high-magnetic field radio pulsars (Mignani et al. 2007b) and the XDINSs (Lo Curto et al. 2007) but, so far, with unconclusive results. Evidence for a fallback disk has been possibly found in Spitzer observations of the AXP 4U 0142+61 (Wang et al. 2006). We note that the $F_{\mathrm{IR}} / F_{\mathrm{X}}$ ratio of the Vela Jr. $\mathrm{CCO}$ is $\sim 5 \times 10^{-4}$, which is very similar to the value observed for the case of AXP 4 U 0142+61. Accurate multi-band IR photometry is required to test such an hypothesis.

The last possibility is that object A is the stellar mass companion of the CCO. We have tried to determine a possible stellar classification of object A by using the available color information. Unfortunately, its non-detection in the $J$-band leaves us with only a measure of $H-K_{\mathrm{s}}$ and with upper limits on $J-H$ and $J-K_{\mathrm{s}}$. However, the fact that object A has not been detected in the $J$-band but only in the $H$ and $K_{\mathrm{s}}$-bands, despite similarly deep exposures, seems to imply that its spectrum is quite red. To better evaluate its colors, we compared its 


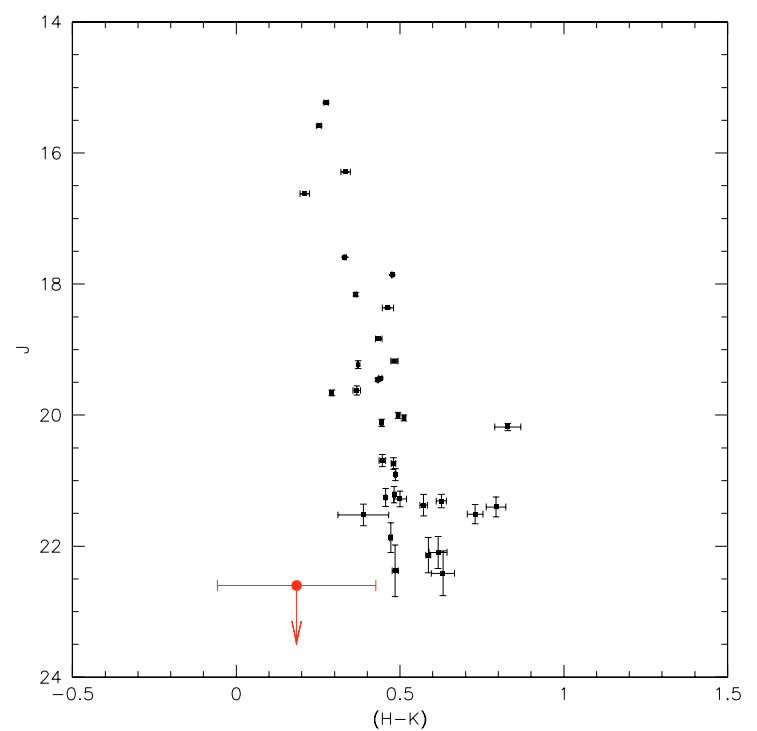

Fig. 3. (Left) $J$ vs. $H-K_{\mathrm{s}}$ color magnitude diagram built for all objects detected in the $\approx 30^{\prime \prime} \times 30^{\prime \prime}$ field around the Vela Junior CCO. Field objects are marked by filled black squares. The possible counterpart to the CCO is marked by the filled red circle. The horizontal bar corresponds to the possible $H-K_{\mathrm{s}}$ range derived from the attached photometry errors.

location in a $J$ vs. $H-K_{\mathrm{s}}$ color magnitude diagram (CMD) with respect to those of a number of stars detected in the field (Fig. 3a). Photometry of the field stars was computed as described in Sect. 3.3. Unfortunately, only $\sim 50$ stars were identified in the narrow and sparsely populated NACO field of view, which certainly hampers our characterization of the field star sequence. At a first glance, with an $H-K_{\mathrm{s}} \sim 0.2$, object A indeed seems to deviate from the sequence. However, its large $H-K_{\mathrm{s}}$ error makes this deviation not significant. Thus, object A is not redder, possibly only slightly bluer, than the majority of the field stars. This can be visually appreciated from the composite $J H K_{\mathrm{s}}$-band image of the field (Fig. 3b). We investigated the effects of the interstellar extinction on the CMD. The interstellar reddening towards the Vela Jr. CCO can be estimated from the Hydrogen column density $N_{\mathrm{H}} \approx 3.5 \times 10^{21} \mathrm{~cm}^{-2}$ derived from the X-ray observations (Kargaltsev et al. 2002). According to the relation of Predhel \& Schmitt (1995) this gives an $E(B-V) \sim 0.67$ which corresponds to $A_{J} \sim 0.5, A_{H} \sim 0.3$ and $A_{K} \sim 0.2$ assuming the extinction coefficients of Fitzpatrick (1999). However, accounting for the reddening does not significantly affect the location of object $\mathrm{A}$ in the $\mathrm{CMD}$ with respect to the sequence of the field stars. Thus, we conclude that object A has no peculiar colors and does not stand out from the average stellar population in the field.

The observed colors of object A, $H-K_{\mathrm{S}}=0.2 \pm 0.2$, $J-H \geq 1.0$, and $R-K_{\mathrm{s}} \geq 5.2$ can be compatible with those of a mid M-type star, after accounting for the interstellar reddeding (e.g. Legget 1992). For instance, an M5 star at a distance of $2 \mathrm{kpc}$ would be just below our detection limit in $J$ but would have $H$ and $K_{\mathrm{s}}$-band magnitudes consistent with those measured for object A. We note that a distance of $2 \mathrm{kpc}$ is larger than that of $1 \mathrm{kpc}$ usually assumed for the SNR (Slane et al. 2001), but is not incompatible with the observations. Thus, our data are consistent with the Vela Jr. CCO being a neutron star in a binary system with an M-type companion star. However, since we have only a lower limit on $J-H$, we can not exclude that object A is actually a star of an even later spectral-type, an example of the recently discovered L dwarfs (Kirkpatrick et al. 1999). Latetype star counterparts have been also proposed in the past for the PKS 1209-52 (Pavlov et al. 2004) and RCW103 (Sanwal et al. 2002) CCOs but both identifications were later discarded from a re-analysis of the Chandra astrometry (Mignani et al. 2007b; Wang et al. 2007; De Luca et al. private communication). If our identification is confirmed, the association of a young neutron star in a binary system with such a low mass companion will pose more than one theoretical challenge for current neutron star formation and supernova explosion models.

\section{Conclusions}

We have reported optical/IR VLT observations of the Vela Jr. CCO. The VLT $R$-band data revealed a compact nebula at the Chandra position of the CCO, consistent both in morphology and in brightness with the $\mathrm{H}_{\alpha}$ nebula identified by Pellizzoni et al. (2002). We can not presently determine whether the nebula is associated with a bow-shock produced by the supersonic motion of the neutron star in the ISM, or if it is a photo-ionization nebula produced by the UV thermal radiation from the neutron star. Higher resolution imaging is required to resolve the nebula structure and to determine whether it is more compatible with the bow-shock or the photo-ionization scenario, for which we expect quite distinct morphologies, i.e. arc-like or spherical, respectively. Alternatively, high-resolution spectroscopy would make it possible to measure the width of the $\mathrm{H}_{\alpha}$ line and to derive its velocity broadening which is typically higher for a velocity driven bow-shock nebula.

Proper motion measurements of the $\mathrm{CCO}$ also represent a powerful diagnostic to investigate the bow-shock scenario, where we expect the velocity vector to lie along the symmetry axis of the nebula and to point towards its apex. Since the CCO is radio-quiet, one possibility for obtaining a proper motion measurement would be through high resolution X-ray observations with Chandra. However, proper motion measurement performed with Chandra on some nearby INSs (Motch et al. 2007) only yiielded marginally significant results. For the Vela Jr. CCO, which is up to a factor five more distant, such measurements are even more difficult. More realistically, a proper motion measurement could be obtained from high-resolution IR imaging of the candidate CCO counterpart identified in our VLT $H$ and $K_{\mathrm{s}}$-band observations. Its proper motion measurement will allow testing of the bow-shock interpretation for the nebula on one hand, and confirming its proposed identification, which only relies on the coincidence with the Chandra position, on the other.

The nature of the candidate $\mathrm{CCO}$ counterpart is, at present, unclear. It could be the neutron star itself, or a fallback disk left over from the supernova explosion, or a very late-type star in a binary system with the neutron star. In all cases, confirming the identification will have several important implications for our understanding of the CCOs as a class, as well as for neutron star formation and supernova explosion models. Instead, discarding the proposed identification will strengthen the more standard framework isolated neutron star scenario for the CCOs.

Acknowledgements. R.P.M. warmly thanks N. Ageorges (ESO) for her invaluable help and friendly support at the telescope, and D. Dobrzycka (ESO) for reducing the IR data with the $N A C O$ pipeline.

\section{References}

Aschenbach, B. 1998, Nature, 396, 141

Aschenbach, B., Egger, R., \& Trümper, J. 1995, Nature, 373, 587

Caraveo, P. A., Bignami, G. F., \& Trümper, J. 1996, A\&AR, 7, 209 
Caraveo, P. A., Mignani, R. P., DeLuca, A., \& Wagner, S. 2001, Proc. of A decade of HST science, STScI 105, 9 [arXiv:astro-ph/0009035]

Fitzpatrick, E. L. 1999, PASP, 111, 63

Fesen, R. A., Pavlov, G. G., \& Sanwal, D. 2006, ApJ, 636, 848

Haberl, F. 2005, Proc. of EPIC XMM-Newton Consortium Meeting, MPE Report, 288, 39

Hester, J. J., Scowen, P. A., Sankrit, R., et al. 1995, ApJ, 448, 240

Israel, G. L., Covino, S., Perna, R., et al. 2003, ApJ, 589, L93

Israel, G. L., Rea, N., Mangano, V., et al. 2004, ApJ, 603, L97

Kargaltsev, O., Pavlov, G. G., Sanwal, D., \& Garmire, G. P. 2002, ApJ, 580, 1060

Kirkpatrick, J. D., Reid, I. N., Liebert, J., et al. 1999, ApJ, 519,802

Landolt, A. U. 1992, ApJS, 82, 351

Legget, S. K. 1992, AJ, 104, 340

Lo Curto, G., Mignani, R. P., Perna, R., \& Israel, G. L. 2007, A\&A, in press [arXiv.0706.2573]

Mereghetti, S. 2001, ApJ, 548, L213

Mereghetti, S., Pellizzoni, A., \& de Luca, A. 2002, Proc. of Neutron Stars in Supernova Remnants, ASP Conf. Ser., 271, 289

Michael, E. 2000, ApJS, 127, 429

Mignani, R. P., Bagnulo, S., De Luca, A., et al. 2007a, Ap\&SS, 308, 203

Mignani, R. P., Perna, R., Rea, N., et al. 2007b, A\&A, 471, 265

Motch, C., Pires, A. M., Haberl, F., \& Schwope, A. 2007, Ap\&SS, 308, 217

Parker, Q. A., \& Phillips, S. 1998, PASA, 15, 28
Pavlov, G. G., Sanwal, D., Kiziltan, B., \& Garmire, G. P. 2001, ApJ, 559, L131 Pavlov, G. G., Sanwal, D., \& Teter, M. A. 2004, Proc. of Young Neutron Stars and Their Environments (ASP), 239

Pellizzoni, A., Mereghetti, S., \& De Luca, A. 2002, A\&A, 393, L65

Persson, S. E., Murphy, D. C., Krzeminski, W., Roth, M., \& Rieke, M. J. 1998, AJ, 116, 2475

Predehl, P., \& Schmitt, J. H. M. M. 1995, A\&A, 293, 889

Reynoso, E. M., Dubner, G., Giacani, E., Johnston, S., \& Green, A. J. 2006, A\&A, 449, 243

Sanwal, D., Garmire, G. P., Garmire, A., Pavlov, G. G., \& Mignani, R. 2002, AAS, 34, 764

Slane, P., Hughes, J. P., Edgar, R. J., et al. 2001, ApJ, 548, 814

Stappers, B. W., Gaensler, B. M., Kaspi, V. M., van der Klis, M., \& Lewin, W. H. G. 2003, Science, 299, 1372

Stetson, P. B. 1992, Astronomical Data Analysis Software and Systems I, ASP Conf. Ser., 25, 297

Stetson, P. B. 1994, Calibrating Hubble Space Telescope, 89

van Kerkwijk, M. H., \& Kulkarni, S. R. 2001, A\&A, 380, 221

Wang, Z., Chakrabarty, D., \& Kaplan, D. L. 2006, Nature, 440, 772

Wang, Z., Kaplan, D., \& Chakrabarty, D. 2007, ApJ, 655, 261

Woods, P. M., \& Thompson, C. 2006, in Compact Stellar X-ray Sources, ed. W. H. G. Lewin, \& M. van der Klis, 39, 547

Zaggia, S. R., Piotto, G., \& Capaccioli, M. 1997, A\&A, 327, 1004 